\title{
TRACES OF HECKE OPERATORS IN LEVEL 1 AND GAUSSIAN HYPERGEOMETRIC FUNCTIONS
}

\author{
JENNY G. FUSELIER
}

(Communicated by Ken Ono)

\begin{abstract}
We provide formulas for traces of $p^{\text {th }}$ Hecke operators in level 1 in terms of values of finite field ${ }_{2} F_{1}$-hypergeometric functions, extending previous work of the author to all odd primes $p$ instead of only those $p \equiv 1(\bmod 12)$. We first give a general level 1 trace formula in terms of the trace of Frobenius on a family of elliptic curves, and then we draw on recent work of Lennon to produce level 1 trace formulas in terms of hypergeometric functions for all primes $p>3$.
\end{abstract}

\section{INTRODUCTION}

In recent years, relationships between traces of Hecke operators and counting points on families of varieties have been explored. For example, Ahlgren and Ono [2] described traces of $p^{t h}$ Hecke operators in weight 4 and level 8 in terms of the number of $\mathbb{F}_{p}$-points on a Calabi-Yau threefold, while in [1, Ahlgren related traces of Hecke operators in weight 6 and level 4 to counting $\mathbb{F}_{p}$-points on the Legendre family of elliptic curves. The level 2 formula (for all weights) was made explicit in terms of the number of $\mathbb{F}_{p}$-points on a family of elliptic curves by Frechette, Ono, and Papanikolas in [5]. In [7, the author considered the level 1 case and provided a formula in terms of the number of $\mathbb{F}_{p}$-points on a one-parameter family of elliptic curves for primes $p \equiv 1(\bmod 12)$. Most recently, Lennon [12] considered the levels 3 and 9 scenarios. Earlier work of Ihara [10] and Birch [4] gave reason to believe such formulas were possible.

Interestingly, these trace formulas also have a link to finite field hypergeometric functions introduced by Greene in the 1980s [8]. Various authors [3, 5, 12] have used relations between values of Greene's hypergeometric functions and counting $\mathbb{F}_{p}$-points on varieties to produce trace formulas in terms of hypergeometric functions. In [7, Thm. 1.2], the author proved an explicit relationship between counting $\mathbb{F}_{p}$-points on a one-parameter family of elliptic curves and the values of a particular ${ }_{2} F_{1}$ function over $\mathbb{F}_{p}$, which led to a level 1 trace formula in terms of hypergeometric functions. However, this formula was only proved for primes $p \equiv 1(\bmod 12)$. Recently, Lennon [11, Thms. 1.1 and 2.1] has removed this restriction on the congruence class of $p$ to produce formulas that relate $\# E\left(\mathbb{F}_{q}\right)$ to values of a ${ }_{2} F_{1}$ function over $\mathbb{F}_{q}$ for any $q=p^{e}$ where $q \equiv 1(\bmod 12)$.

In this paper, we provide a level 1 trace formula that holds for all $p>3$. Then, we use Lennon's result to produce formulas for traces of Hecke operators in level 1 in terms of finite field hypergeometric functions.

Received by the editors September 15, 2011.

2010 Mathematics Subject Classification. Primary 11F30; Secondary 11T24, 11G20, 33C99.

(C)2012 American Mathematical Society 1871 


\section{Statement of MAin RESUlts}

Let $p>3$ be prime and let $k \geq 2$ be an even integer. Define $F_{k}(x, y)=\frac{x^{k-1}-y^{k-1}}{x-y}$. Then letting $x+y=s$ and $x y=p$ gives rise to polynomials $G_{k}(s, p)=F_{k}(x, y)$. These polynomials can be written alternatively as

$$
G_{k}(s, p)=\sum_{j=0}^{\frac{k}{2}-1}(-1)^{j}\left(\begin{array}{c}
k-2-j \\
j
\end{array}\right) p^{j} s^{k-2 j-2} .
$$

Throughout, results will depend on the congruence class of $p \bmod 12$. As such, we set up some notation for various congruence classes of $p$ to be used throughout the remainder of the paper. Whenever $p \equiv 1(\bmod 4)$, we let $a, b \in \mathbb{Z}$ be such that $p=a^{2}+b^{2}$ and $a+b i \equiv 1(2+2 i)$ in $\mathbb{Z}[i]$. In that case, we define

$$
\mu_{k}(p)=\frac{1}{2}\left[G_{k}(2 a, p)+G_{k}(2 b, p)\right] .
$$

Similarly, whenever $p \equiv 1(\bmod 3)$, we let $c, d \in \mathbb{Z}$ be such that $p=c^{2}-c d+d^{2}$ and $c+d \omega \equiv 2(3)$ in $\mathbb{Z}[\omega]$, where $\omega=e^{2 \pi i / 3}$. In this case, we define

$$
\nu_{k}(p)=\frac{1}{3}\left[G_{k}(c+d, p)+G_{k}(2 c-d, p)+G_{k}(c-2 d, p)\right] .
$$

We consider a one-parameter family of elliptic curves having $j$-invariant $\frac{1728}{t}$. Specifically, for $t \in \mathbb{F}_{p} \backslash\{0,1\}$, we let

$$
E_{t}: y^{2}=4 x^{3}-\frac{27}{1-t} x-\frac{27}{1-t} .
$$

Let $a(t, p)$ denote the trace of the Frobenius endomorphism on $E_{t}$. In particular, for $t \neq 0,1$, we have

$$
a(t, p)=p+1-\# E_{t}\left(\mathbb{F}_{p}\right) .
$$

Let $\Gamma=S L_{2}(\mathbb{Z})$ and let $M_{k}$ and $S_{k}$, respectively, denote the spaces of modular forms and cusp forms of weight $k$ for $\Gamma$. Further, let $\operatorname{Tr}_{k}(\Gamma, p)$ denote the trace of the Hecke operator $\mathrm{T}_{k}(p)$ on $S_{k}$. Our first main result completely classifies the traces of cusp forms in level 1:

Theorem 2.1. Let $p>3$ be prime. Then for even $k \geq 4$,

$$
\operatorname{Tr}_{k}(\Gamma, p)=-1-\lambda(k, p)-\sum_{t=2}^{p-1} G_{k}(a(t, p), p),
$$

where

$$
\lambda(k, p)=\left\{\begin{array}{lll}
\mu_{k}(p)+\nu_{k}(p) & \text { if } p \equiv 1 \quad(\bmod 12) \\
\mu_{k}(p)+(-p)^{\frac{k}{2}-1} & \text { if } p \equiv 5 \quad(\bmod 12), \\
\nu_{k}(p)+(-p)^{\frac{k}{2}-1} & \text { if } p \equiv 7 \quad(\bmod 12) \\
2(-p)^{\frac{k}{2}-1} & \text { if } p \equiv 11 \quad(\bmod 12)
\end{array}\right.
$$

Next, we move to results which link these traces of Hecke operators in level 1 with hypergeometric functions over finite fields. We begin with some preliminaries. Let $p$ be a prime and let $q=p^{e}$. Let $\widehat{F_{q}^{\times}}$denote the group of all multiplicative characters on $\mathbb{F}_{q}^{\times}$. We extend $\chi \in \widehat{\mathbb{F}}_{q}^{\times}$to all of $\mathbb{F}_{q}$ by setting $\chi(0)=0$. We let $\varepsilon$ 
denote the trivial character. For $A, B \in \widehat{\mathbb{F}_{p}^{\times}}$, let $J(A, B)$ denote the usual Jacobi symbol and define

$$
\left(\begin{array}{l}
A \\
B
\end{array}\right):=\frac{B(-1)}{q} J(A, \bar{B})=\frac{B(-1)}{q} \sum_{x \in \mathbb{F}_{q}} A(x) \bar{B}(1-x)
$$

Greene defined hypergeometric functions over $\mathbb{F}_{q}$ in the following way:

Definition 2.2 ([8], Defn. 3.10). If $n$ is a positive integer, $x \in \mathbb{F}_{q}$, and $A_{0}, A_{1}, \ldots$, $A_{n}, B_{1}, B_{2}, \ldots, B_{n} \in \widehat{\mathbb{F}_{q}^{\times}}$, then define

$$
{ }_{n+1} F_{n}\left(\begin{array}{cccc}
A_{0}, & A_{1}, & \ldots, & A_{n} \\
& B_{1}, & \ldots, & B_{n}
\end{array} \mid x\right)_{q}:=\frac{q}{q-1} \sum_{\chi \in \mathbb{F}_{q}^{\times}}\left(\begin{array}{c}
A_{0} \chi \\
\chi
\end{array}\right)\left(\begin{array}{c}
A_{1} \chi \\
B_{1} \chi
\end{array}\right) \ldots\left(\begin{array}{c}
A_{n} \chi \\
B_{n} \chi
\end{array}\right) \chi(x) .
$$

In 17, Thm. 1.2], the author proved a formula giving an explicit relationship between $a(t, p)$ and a ${ }_{2} F_{1}$-hypergeometric function over $\mathbb{F}_{p}$, but required that $p \equiv 1$ $(\bmod 12)$. In this case, the result of Theorem 2.1 can be rewritten to be in terms of a hypergeometric function over $\mathbb{F}_{p}$. However, 7 did not address the other classes of primes $\bmod 12$. Notice that either $p \equiv 1(\bmod 12)$ or, if not, then $p^{2} \equiv 1(\bmod 12)$. With this in mind, for the remainder of the paper we define $q=p^{e(p)}$, where

$$
e(p)=\left\{\begin{array}{lll}
1 & \text { if } p \equiv 1 & (\bmod 12) \\
2 & \text { if } p \neq \equiv 1 & (\bmod 12)
\end{array}\right.
$$

We consider the same family of elliptic curves $E_{t}$, as defined in (4), but now over $\mathbb{F}_{q}$, and with $a(t, q)=q+1-\# E_{t}\left(\mathbb{F}_{q}\right)$. Thanks to Lennon's results [1] and an inverse pair given in [13, we can now describe the traces of Hecke operators in level 1 in terms of a ${ }_{2} F_{1}$ function over $\mathbb{F}_{q}$ for the other classes of primes mod 12 :

Theorem 2.3. Let $p>3$ be prime such that $p=5,7,11(\bmod 12)$ with $q=p^{e(p)}$ and $T$ a generator of $\widehat{\mathbb{F}_{q}^{x}}$. Let $k \geq 4$ be even and $m=\frac{k}{2}-1$. Define $H_{m}(x):=$ $\sum_{i=0}^{m}\left(\begin{array}{c}m+i \\ m-i\end{array}\right) x^{i}$. Then

$$
\begin{aligned}
\operatorname{Tr}_{k}(\Gamma, p) & =-1-\lambda(k, p) \\
- & \sum_{t=2}^{p-1}(-p)^{m} H_{m}\left(p T^{\frac{q-1}{2}}(2) T^{\frac{q-1}{4}}(1-t)_{2} F_{1}\left(\begin{array}{cc}
T^{\frac{q-1}{12}}, & T^{\frac{5(q-1)}{12}} \\
\varepsilon
\end{array} \mid t\right)_{q}-2\right)
\end{aligned}
$$

where $\lambda(k, p)$ is as in Theorem 2.1 .

Our final result is a generalization of [7, Thm. 1.4], giving a recursive formula for traces of Hecke operators in level 1 in terms of hypergeometric functions, now for all primes $p>3$ :

Theorem 2.4. Let $p>3$ be prime, and $q=p^{e(p)}$. Let $k \geq 4$ be even, and $m=\frac{k}{2}-1$. Further, let $T$ be a generator of $\widehat{\mathbb{F}_{q}^{\times}}$and $b_{i}=p^{m-i}\left[\left(\begin{array}{c}2 m \\ m-i\end{array}\right)-\left(\begin{array}{c}2 m \\ m-i-1\end{array}\right)\right]$. 
Then

$$
\begin{aligned}
\operatorname{Tr}_{2(m+1)}(\Gamma, p)= & -1-\lambda(2 m+2, p)+b_{0}(p-2) \\
& -\sum_{i=1}^{m-1} b_{i} \cdot\left(\operatorname{Tr}_{2 i+2}(\Gamma, p)+1+\lambda(2 i+2, p)\right) \\
& -\sum_{t=2}^{p-1}\left(\psi(t, q)_{2} F_{1}\left(\begin{array}{cc}
T^{\frac{q-1}{12}}, & T^{\frac{5(q-1)}{12}} \\
\varepsilon
\end{array} \mid t\right)_{q}+2 p(e(p)-1)\right)^{\frac{2 m}{e(p)}},
\end{aligned}
$$

where

and $\lambda(k, p)$ is as in Theorem 2.1.

$$
\psi(t, q)=-q T^{\frac{q-1}{2}}(2) T^{\frac{q-1}{4}}(1-t)
$$

\section{Proof of Theorem 2.1}

To prove Theorem 2.1, we begin with Hijikata's version of the Eichler-Selberg trace formula 9]. The statement of this theorem requires some notation. If $d<0$, $d \equiv 0,1(\bmod 4)$, let $\mathcal{O}(d)$ denote the unique imaginary quadratic order in $\mathbb{Q}(\sqrt{d})$ having discriminant $d$. Let $h(d)=h(\mathcal{O}(d))$ be the order of the class group of $\mathcal{O}(d)$, and let $w(d)=w(\mathcal{O}(d))$ be half the cardinality of the unit group of $\mathcal{O}(d)$. We then let $h^{*}(d)=h(d) / w(d)$. The following theorem is the level 1 formulation of Hijikata's version of the Eichler-Selberg trace formula for any odd prime.

Theorem 3.1. Let $p$ be an odd prime and $k \geq 2$ be even. Then

$$
\operatorname{Tr}_{k}(\Gamma, p)=-1-\frac{1}{2} \beta(p)(-p)^{\frac{k}{2}-1}-\sum_{0<s<2 \sqrt{p}} G_{k}(s, p) \sum_{f \mid \ell} h^{*}\left(\frac{s^{2}-4 p}{f^{2}}\right)+\delta(k),
$$

where

$$
\beta(p)= \begin{cases}h^{*}(-4 p) & \text { if } p \equiv 1 \quad(\bmod 4), \\ h^{*}(-4 p)+h^{*}(-p) & \text { if } p \equiv 3 \quad(\bmod 4),\end{cases}
$$

$\delta(k)=p+1$ if $k=2$ and 0 otherwise, and where we classify integers $s$ with $s^{2}-4 p<0$ by some positive integer $\ell$ and square-free integer $m$ via

$$
s^{2}-4 p= \begin{cases}\ell^{2} m, & 0>m \equiv 1 \quad(\bmod 4) \\ \ell^{2} 4 m, & 0>m \equiv 2,3 \quad(\bmod 4) .\end{cases}
$$

To link Theorem 3.1 to $a(t, p)$, we need to consider all isomorphism classes of elliptic curves over $\mathbb{F}_{p}$. If $E$ is any elliptic curve defined over $\mathbb{F}_{p}$, let $a(E)=$ $p+1-\# E\left(\mathbb{F}_{p}\right)$. Additionally, for a perfect field $K$, we define

$$
E l l_{K}:=\left\{[E]_{K}: E \text { is defined over } K\right\},
$$

where $[E]_{K}$ denotes the isomorphism class of $E$ over $K$ and $\left[E_{1}\right]_{K}=\left[E_{2}\right]_{K}$ if there exists an isomorphism $\beta: E_{1} \rightarrow E_{2}$ over $K$. We first address the cases $j(E)=1728$ and $j(E)=0$.

Lemma 3.2. Let $p$ be an odd prime. Whenever $p \equiv 1(\bmod 4)$, define $a, b \in \mathbb{Z}$ to be such that $p=a^{2}+b^{2}$ and $a+b i \equiv 1(2+2 i)$ in $\mathbb{Z}[i]$. Then, for $n \geq 2$ even,

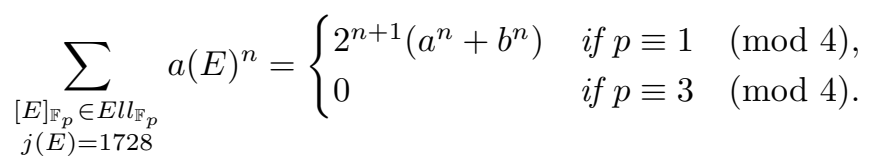


Proof. The case $p \equiv 1(\bmod 4)$ was proved by the author in [6, Lemma IV.3.3]. If $p \equiv 3(\bmod 4)$ and $[E]_{\mathbb{F}_{p}} \in E l l_{\mathbb{F}_{p}}$, then $\# E\left(\mathbb{F}_{p}\right)=p+1$, so $a(E)=0$.

Lemma 3.3. Let $p>3$ be prime. Whenever $p \equiv 1(\bmod 3)$, we let $c, d \in \mathbb{Z}$ be such that $p=c^{2}-c d+d^{2}$ and $c+d \omega \equiv 2(3)$ in $\mathbb{Z}[\omega]$, where $\omega=e^{2 \pi i / 3}$. Then, for $n \geq 2$ even,

$$
\sum_{\substack{[E]_{\mathbb{F}_{p}} \in E l l_{\mathbb{F}_{p}} \\
j(E)=0}} a(E)^{n}=\left\{\begin{array}{lll}
2\left[(c+d)^{n}+(2 c-d)^{n}+(c-2 d)^{n}\right] & \text { if } p \equiv 1 & (\bmod 3), \\
0 & \text { if } p \equiv 2 & (\bmod 3) .
\end{array}\right.
$$

Proof. The case $p \equiv 1(\bmod 3)$ was proved by the author in [6, Lemma IV.3.5]. If $p \equiv 2(\bmod 3)$ and $[E]_{\mathbb{F}_{p}} \in E l l_{\mathbb{F}_{p}}$, then $\# E\left(\mathbb{F}_{p}\right)=p+1$, so $a(E)=0$.

The proof of Theorem 2.1 proceeds along the same line as the proof of the $p \equiv 1$ ( $\bmod 12)$ case proved by the author in 7 . In particular, we begin with the following extension of [7, Lemma 5.3].

Lemma 3.4. Let $p>3$ be prime. Then for $n \geq 2$ even,

$$
\begin{aligned}
\sum_{0<s<2 \sqrt{p}} s^{n} \sum_{f \mid \ell} h\left(\frac{s^{2}-4 p}{f^{2}}\right)= & \sum_{0<s<2 \sqrt{p}} s^{n} \sum_{f \mid \ell} h^{*}\left(\frac{s^{2}-4 p}{f^{2}}\right) \\
& +\frac{1}{4} \sum_{\substack{[E]_{\mathbb{F}_{p}} \in E l l_{\mathbb{F}_{p}} \\
j(E)=1728}} a(E)^{n}+\frac{1}{3} \sum_{\substack{[E]_{\mathbb{F}_{p}} \in E l l_{\mathbb{F}_{p}} \\
j(E)=0}} a(E)^{n} .
\end{aligned}
$$

Proof. The proof for primes $p \equiv 1(\bmod 12)$ is provided in [7, Lemma 5.3]. It can be adapted to hold for all $p>3$ once one verifies that the following two identities remain true:

$$
\begin{gathered}
\sum_{0<s<2 \sqrt{p}} s^{n} \sum_{\substack{f \mid \ell \\
\frac{s^{2}-4 p}{f^{2}}=-4}} 1=\frac{1}{2} \sum_{\substack{[E]_{\mathbb{F} p} \in E l l_{\mathbb{F}_{p}} \\
j(E)=1728}} a(E)^{n}, \\
\sum_{0<s<2 \sqrt{p}} s^{n} \sum_{\frac{s^{2}-4 p}{f^{2}}=-3} 1=\frac{1}{2} \sum_{\substack{[E]_{\mathbb{F}_{p}} \in E l l_{\mathbb{F}} \\
j(E)=0}} a(E)^{n} .
\end{gathered}
$$

First consider (7). The proof given in [7] holds for all primes $p \equiv 1(\bmod 4)$. In light of Lemma 3.2, we must verify that if $p \equiv 3(\bmod 4)$, then

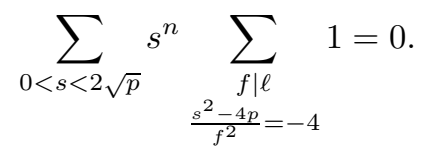

We verify this by proving that no $s, f$ exist to contribute to the sums. For, suppose $s, f \in \mathbb{Z}$ such that $0<s<2 \sqrt{p}$ and $\frac{s^{2}-4 p}{f^{2}}=-4$. Then $4 \mid s^{2}$, so $s$ must be even. Substituting $s=2 r$ and rearranging gives $r^{2}+f^{2}=p$, which is not possible since $p \equiv 3(\bmod 4)$. This verifies (17) for the remaining primes. 
We handle (8) in a similar way. The proof in [7] verifies the equation for $p \equiv 1$ $(\bmod 3)$. Keeping in mind Lemma 3.3 , we must prove that if $p \equiv 2(\bmod 3)$, then

$$
\sum_{0<s<2 \sqrt{p}} s^{n} \sum_{\substack{f \mid \ell \\ s^{2}-4 p}} 1=-3
$$

Suppose then that we have $s, f \in \mathbb{Z}$ such that $0<s<2 \sqrt{p}$ and $\frac{s^{2}-4 p}{f^{2}}=-3$. Then $4 p=3 f^{2}+s^{2}$ and hence $p \equiv 2 \equiv s^{2}(\bmod 3)$. However, since $\left(\frac{2}{3}\right)=-1$, this is impossible. This verifies (8) for the remaining primes and completes the proof of the lemma.

The following proposition generalizes [7, Prop. 5.4] by removing the restriction on the congruence class of $p(\bmod 12)$.

Proposition 3.5. Let $p>3$ be prime and $n \geq 2$ be even. Then

$$
\sum_{t=2}^{p-1} a(t, p)^{n}=\sum_{0<s<2 \sqrt{p}} s^{n} \sum_{f \mid \ell} h^{*}\left(\frac{s^{2}-4 p}{f^{2}}\right)-\alpha(n, p)-\gamma(n, p),
$$

where

$$
\begin{aligned}
& \alpha(n, p)=\left\{\begin{array}{lll}
2^{n-1}\left(a^{n}+b^{n}\right) & \text { if } p \equiv 1 \quad(\bmod 4), \\
0 & \text { if } p \equiv 3 \quad(\bmod 4),
\end{array}\right. \\
& \gamma(n, p)\left\{\begin{array}{lll}
\frac{1}{3}\left[(c+d)^{n}+(2 c-d)^{n}+(c-2 d)^{n}\right] & \text { if } p \equiv 1 & (\bmod 3), \\
0 & \text { if } p \equiv 2 & (\bmod 3),
\end{array}\right.
\end{aligned}
$$

and $a, b, c, d$ are defined as in the statements of Lemmas 3.2 and 3.3 .

Proof. The bulk of the proof the author provides for the $p \equiv 1(\bmod 12)$ case in 7. Prop. 5.4] holds for the other congruence classes of $p$, so we give an outline here. Since $j\left(E_{t}\right)=\frac{1728}{t}$, we have that

$$
\begin{aligned}
\sum_{t=2}^{p-1} a(t, p)^{n} & =\sum_{\substack{[E]_{\mathbb{F}_{p}} \in E l l_{\mathbb{F}_{p}} ; E / \mathbb{F}_{p} \\
j(E) \neq 0,1728}} a(E)^{n}=\frac{1}{2} \sum_{\substack{[E]_{\mathbb{F}_{p}} \in E l l_{\mathbb{F}_{p}} \\
j(E) \neq 0,1728}} a(E)^{n} \\
& =\frac{1}{2}\left[\sum_{[E]_{\mathbb{F}_{p}} \in E l l_{\mathbb{F}_{p}}} a(E)^{n}-\sum_{\substack{[E]_{\mathbb{F}_{p}} \in E l l_{\mathbb{F}_{p}} \\
j(E)=1728}} a(E)^{n}-\sum_{\substack{[E]_{\mathbb{F}_{p}} \in E l l_{\mathbb{F}_{p}} \\
j(E)=0}} a(E)^{n}\right] .
\end{aligned}
$$

Regardless of the congruence class of $p(\bmod 12)$, the first sum in the last line above can still be written in terms of class numbers by combining Hasse's theorem with a theorem of Schoof [14, Thm. 4.6]. This results in

$$
\begin{aligned}
\sum_{t=2}^{p-1} a(t, p)^{n} & =\sum_{0<s<2 \sqrt{p}} s^{n} \sum_{f \mid \ell} h\left(\frac{s^{2}-4 p}{f^{2}}\right)-\frac{1}{2} \sum_{\substack{[E]_{\mathbb{F}_{p}} \in E l l_{\mathbb{F}_{p}} \\
j(E)=1728}} a(E)^{n}-\frac{1}{2} \sum_{\substack{[E]_{\mathbb{F}_{p}} \in E l l_{\mathbb{F}_{p}} \\
j(E)=0}} a(E)^{n} \\
& =\sum_{0<s<2 \sqrt{p}} s^{n} \sum_{f \mid \ell} h^{*}\left(\frac{s^{2}-4 p}{f^{2}}\right)-\frac{1}{4} \sum_{\substack{[E]_{\mathbb{F}_{p}} \in E l_{\mathbb{F}_{p}} \\
j(E)=1728}} a(E)^{n}-\frac{1}{6} \sum_{\substack{[E]_{\mathbb{F}_{p}} \in E l l_{\mathbb{F}_{p}} \\
j(E)=0}} a(E)^{n}
\end{aligned}
$$


by Lemma 3.4. One now applies Lemmas 3.2 and 3.3 in each appropriate congruence class to obtain the result.

With these tools in place, we now complete the proof of Theorem 2.1

Proof of Theorem 2.1. The proof proceeds in a similar fashion to the author's proof of the $p \equiv 1(\bmod 12)$ case in 7 , Thm. 1.3], with a few modifications. We still begin with an application of Theorem 3.1 and then substitute the definition of $G_{k}(s, p)$. This gives

$$
\text { (9) } \begin{aligned}
\operatorname{Tr}_{k}(\Gamma, p)=- & -\frac{1}{2} \beta(p)(-p)^{\frac{k}{2}-1}-(-p)^{\frac{k}{2}-1} \sum_{0<s<2 \sqrt{p}} 1 \sum_{f \mid \ell} h^{*}\left(\frac{s^{2}-4 p}{f^{2}}\right) \\
& -\sum_{j=0}^{\frac{k}{2}-2}(-1)^{j}\left(\begin{array}{c}
k-2-j \\
j
\end{array}\right) p^{j} \sum_{0<s<2 \sqrt{p}} s^{k-2 j-2} \sum_{f \mid \ell} h^{*}\left(\frac{s^{2}-4 p}{f^{2}}\right) .
\end{aligned}
$$

Now, notice that the $k=2$ case of Theorem 3.1 gives

$$
0=p-\frac{1}{2} \beta(p)-\sum_{0<s<2 \sqrt{p}} 1 \sum_{f \mid \ell} h^{*}\left(\frac{s^{2}-4 p}{f^{2}}\right) .
$$

Substituting and applying Proposition 3.5 with $n=k-2 j-2$ gives

$$
\begin{aligned}
\operatorname{Tr}_{k}(\Gamma, p)= & -1+(-p)^{\frac{k}{2}-1} \cdot(-p)-\sum_{j=0}^{\frac{k}{2}-2}(-1)^{j}\left(\begin{array}{c}
k-2-j \\
j
\end{array}\right) p^{j} \sum_{t=2}^{p-1} a(t, p)^{k-2 j-2} \\
& -\sum_{j=0}^{\frac{k}{2}-2}(-1)^{j}\left(\begin{array}{c}
k-2-j \\
j
\end{array}\right) p^{j} \alpha(k-2 j-2, p) \\
& -\sum_{j=0}^{\frac{k}{2}-2}(-1)^{j}\left(\begin{array}{c}
k-2-j \\
j
\end{array}\right) p^{j} \gamma(k-2 j-2, p) .
\end{aligned}
$$

To complete the proof, we distribute the copies of $(-p)^{\frac{k}{2}-1}$ to the three summations in a specific way. Notice that

$$
(-p)^{\frac{k}{2}-1}(-p)=-(-p)^{\frac{k}{2}-1}(p-2)-(-p)^{\frac{k}{2}-1}-(-p)^{\frac{k}{2}-1} .
$$

First, since $G_{2}=1$, we see that

$$
-(-p)^{\frac{k}{2}-1}(p-2)-\sum_{j=0}^{\frac{k}{2}-2}(-1)^{j}\left(\begin{array}{c}
k-2-j \\
j
\end{array}\right) p^{j} \sum_{t=2}^{p-1} a(t, p)^{k-2 j-2}=-\sum_{t=2}^{p-1} G_{k}(a(t, p), p) .
$$

A straightforward calculation for each of the congruence classes of $p(\bmod 12)$ verifies

$$
\begin{aligned}
\lambda(k, p)= & (-p)^{\frac{k}{2}-1}+\sum_{j=0}^{\frac{k}{2}-2}(-1)^{j}\left(\begin{array}{c}
k-2-j \\
j
\end{array}\right) p^{j} \alpha(k-2 j-2, p) \\
& +(-p)^{\frac{k}{2}-1}+\sum_{j=0}^{\frac{k}{2}-2}(-1)^{j}\left(\begin{array}{c}
k-2-j \\
j
\end{array}\right) p^{j} \gamma(k-2 j-2, p) .
\end{aligned}
$$




\section{TRACE FORMULAS IN TERMS OF HYPERGEOMETRIC FUNCTIONS}

We now prove Theorems 2.3 and 2.4. As mentioned before, one essential tool is a theorem of Lennon, which writes the trace of Frobenius of any elliptic curve in Weierstrass form in terms of a finite field hypergeometric function:

Theorem 4.1 ([11, Thm. 2.1]). Let $q=p^{e}$, where $p>3$ is prime and $q \equiv 1$ $(\bmod 12)$. Let $E: y^{2}=x^{3}+a x+b$ be an elliptic curve over $\mathbb{F}_{q}$ in Weierstrass form with $j(E) \neq 0,1728$. Then the trace of the Frobenius map on $E$ can be expressed as

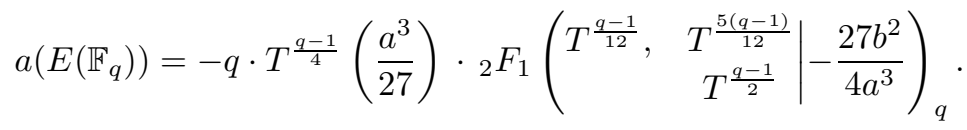

We now specify this theorem to our family of curves.

Corollary 4.2. Let $p>3$ be prime and $q=p^{e(p)}$, where $e(p)$ is defined as in (6). Then

$$
a(t, q)=-q T^{\frac{q-1}{2}}(2) T^{\frac{q-1}{4}}(1-t)_{2} F_{1}\left(\begin{array}{cc}
T^{\frac{q-1}{12}}, & T^{\frac{5(q-1)}{12}} \\
\varepsilon
\end{array} \mid t\right)_{q} .
$$

To prove this corollary, we require a transformation law proved by Greene:

Theorem 4.3 ([8, Thm 4.4(i)]). If $A, B, C \in \widehat{\mathbb{F}}_{q}^{\times}$and $x \in \mathbb{F}_{q} \backslash\{0,1\}$, then

$$
{ }_{2} F_{1}\left(\begin{array}{cc}
A, & B \\
& C
\end{array} \mid x\right)_{q}=A(-1)_{2} F_{1}\left(\begin{array}{cc}
A, & B \\
& A B \bar{C} \mid 1-x
\end{array}\right)_{q} .
$$

Proof of Corollary 4.2. After putting $E_{t}$ into Weierstrass form, we have $a=b=$ $\frac{-27}{4(1-t)}$ in Theorem 4.1. Then $\frac{a^{3}}{27}=\frac{-3^{6}}{4^{3}(1-t)^{3}}$ and $-\frac{27 b^{2}}{4 a^{3}}=1-t$. Combining these simplifications with Greene's theorem above gives

$$
a(t, q)=-q T^{\frac{q-1}{4}}\left(\frac{-3^{6}}{4^{3}(1-t)^{3}}\right) T^{\frac{q-1}{12}}(-1)_{2} F_{1}\left(\begin{array}{cc}
T^{\frac{q-1}{12}} & T^{\frac{5(q-1)}{12}} \\
\varepsilon
\end{array} \mid t\right)_{q} .
$$

Now, using multiplicativity and the fact that $T$ has order $q-1$, we have

$$
\begin{aligned}
T^{\frac{q-1}{4}}\left(\frac{-3^{6}}{4^{3}(1-t)^{3}}\right) & =T^{\frac{3(q-1)}{4}}\left(\frac{-9}{4(1-t)}\right)=T^{\frac{q-1}{4}}\left(\frac{4(1-t)}{-9}\right) \\
& =T^{\frac{q-1}{2}}(2) T^{\frac{q-1}{4}}(1-t) T^{\frac{q-1}{4}}(-1) T^{\frac{q-1}{2}}(3) \\
& =T^{\frac{q-1}{2}}(2) T^{\frac{q-1}{4}}(1-t) T^{\frac{q-1}{4}}(-1),
\end{aligned}
$$

since $T^{\frac{q-1}{2}}$ is its own inverse and $q \equiv 1(\bmod 12)$. The proof is completed by making this substitution for $T^{\frac{q-1}{4}}\left(\frac{-3^{6}}{4^{3}(1-t)^{3}}\right)$ into (12) and noting that $T^{\frac{q-1}{4}}(-1) T^{\frac{q-1}{12}}(-1)$ $=T^{\frac{q-1}{3}}(-1)=1$, since $-1=(-1)^{3}$ and $T^{\frac{q-1}{3}}$ has order 3 .

Remark 4.4. If $e(p)=1$ (i.e. $q=p$ ), the above corollary precisely matches the author's result [7, Thm. 1.3].

Remark 4.5. Lennon gives another way of writing the trace of Frobenius in terms of a ${ }_{2} F_{1}$ function in [11, Thm. 1.1], using the $j$-invariant and discriminant of $E$. We use Lennon's Theorem 2.1 because it leads to a simpler hypergeometric function in this instance. 
We require two more tools to prove our trace theorems. First, note that whenever $e(p)=2$ (i.e. $q=p^{2}$ ), Theorem 4.1 relates $a\left(t, p^{2}\right)$ to a hypergeometric function over $\mathbb{F}_{p^{2}}$. Even though our trace formula, Theorem 2.1 , is in terms of $a(t, p)$, we can still gain new information, since

$$
a(t, p)^{2}=a\left(t, p^{2}\right)+2 p .
$$

The last tool is an inverse pair given in 13 . As in the statement of Theorems 2.3 and 2.4, we let $m=\frac{k}{2}-1$ and also define $H_{m}(x):=\sum_{i=0}^{m}\left(\begin{array}{c}m+i \\ m-i\end{array}\right) x^{i}$. Then, as in [7], notice that

$$
G_{k}(s, p)=(-p)^{m} H_{m}\left(\frac{-s^{2}}{p}\right) .
$$

Consider the inverse pair [13, p. 67] given by

$$
\rho_{n}(x)=\sum_{k=0}^{n}\left(\begin{array}{l}
n+k \\
n-k
\end{array}\right) x^{k}, x^{n}=\sum_{k=0}^{n}(-1)^{k+n}\left[\left(\begin{array}{c}
2 n \\
n-k
\end{array}\right)-\left(\begin{array}{c}
2 n \\
n-k-1
\end{array}\right)\right] \rho_{k}(x) .
$$

Applying this to the definition of $H_{m}$, we see that

$$
x^{m}=\sum_{i=0}^{m}(-1)^{i+m}\left[\left(\begin{array}{c}
2 m \\
m-i
\end{array}\right)-\left(\begin{array}{c}
2 m \\
m-i-1
\end{array}\right)\right] H_{i}(x) .
$$

By combining (14) with the choice $x=\frac{-s^{2}}{p}$, we have

$$
s^{2 m}=\sum_{i=0}^{m} b_{i} G_{2 i+2}(s, p),
$$

where $b_{i}=p^{m-i}\left[\left(\begin{array}{c}2 m \\ m-i\end{array}\right)-\left(\begin{array}{c}2 m \\ m-i-1\end{array}\right)\right]$. We may now prove Theorems 2.3 and 2.4 ,

Proof of Theorem 2.3. Recall that in the statement of this theorem, $p \equiv 5,7,11$ $(\bmod 12)$, so $q=p^{2}$. By (14) and (13), we have

$$
\begin{aligned}
G_{k}(a(t, p), p) & =(-p)^{m} H_{m}\left(\frac{-a(t, p)^{2}}{p}\right) \\
& =(-p)^{m} H_{m}\left(\frac{-a\left(t, p^{2}\right)}{p}-2\right) \\
& =(-p)^{m} H_{m}\left(p T^{\frac{q-1}{2}}(2) T^{\frac{q-1}{4}}(1-t)_{2} F_{1}\left(\begin{array}{cc}
T^{\frac{q-1}{12}}, & T^{\frac{5(q-1)}{12}} \\
\varepsilon
\end{array}\right)_{q}-2\right),
\end{aligned}
$$

by Corollary 4.2. Combining this with Theorem 2.1 completes the proof.

Proof of Theorem 2.4. Recall that $p>3$ is any prime and $q=p^{e(p)}$, where $e(p)$ is defined as in (6). The proof begins along the same lines as the proof of [7, Thm. 1.4]. Beginning with Theorem 2.1, we have

$$
\operatorname{Tr}_{2(m+1)}(\Gamma, p)=-1-\lambda(2 m+2, p)-\sum_{t=2}^{p-1} G_{2 m+2}(a(t, p), p) .
$$

Now, (16) implies that

$$
s^{2 m}=\sum_{i=0}^{m} b_{i} G_{2 i+2}(s, p)=G_{2 m+2}(s, p)+\sum_{i=0}^{m-1} b_{i} G_{2 i+2}(s, p) .
$$


We isolate $G_{2 m+2}(s, p)$ and take $s=a(t, p)$. Substituting into (17) gives

$$
\begin{aligned}
\operatorname{Tr}_{2(m+1)}(\Gamma, p)= & -1-\lambda(2 m+2, p)-\sum_{t=2}^{p-1}\left(a(t, p)^{2 m}-\sum_{i=0}^{m-1} b_{i} G_{2 i+2}(a(t, p), p)\right) \\
= & -1-\lambda(2 m+2, p)-\sum_{t=2}^{p-1} a(t, p)^{2 m}+b_{0}(p-2) \\
& +\sum_{i=1}^{m-1} b_{i} \sum_{t=2}^{p-1} G_{2 i+2}(a(t, p), p) \\
= & -1-\lambda(2 m+2, p)-\sum_{t=2}^{p-1} a(t, p)^{2 m}+b_{0}(p-2) \\
& -\sum_{i=1}^{m-1} b_{i}\left(\operatorname{Tr}_{2 i+2}(\Gamma, p)+1+\lambda(2 i+2, p)\right),
\end{aligned}
$$

since $G_{2}=1$ and by again applying Theorem 2.1 for the last equality. To complete the proof, we consider $a(t, p)^{2 m}$. If $e(p)=1$ (i.e. $q=p$ ), then using either Corollary 4.2 or [7, Thm. 1.2], we have

$$
a(t, p)^{2 m}=\left(-q T^{\frac{q-1}{2}}(2) T^{\frac{q-1}{4}}(1-t)_{2} F_{1}\left(\begin{array}{cc}
T^{\frac{q-1}{12}}, & T^{\frac{5(q-1)}{12}} \\
\varepsilon
\end{array} \mid t\right)_{q}\right)^{2 m},
$$

so our result matches [7, Thm. 1.4] in this case.

If $e(p)=2$ (i.e. $q=p^{2}$ ), then (13) gives

$$
\begin{aligned}
& a(t, p)^{2 m}=\left(a(t, p)^{2}\right)^{m}=\left(a\left(t, p^{2}\right)+2 p\right)^{m}
\end{aligned}
$$

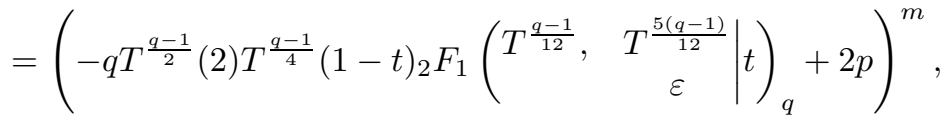

by Corollary 4.2 .

The final statement of the theorem combines these two cases together, completing our proof.

\section{REFERENCES}

[1] S. Ahlgren, The points of a certain fivefold over finite fields and the twelfth power of the eta function, Finite Fields Appl. 8 (2002), no. 1, 18-33. MR.1872789 (2002h:11056)

[2] S. Ahlgren and K. Ono, Modularity of a certain Calabi-Yau threefold, Monatsh. Math. 129 (2000), no. 3, 177-190. MR:1746757(2001b:11059)

[3] S. Ahlgren and K. Ono, A Gaussian hypergeometric series evaluation and Apéry number congruences, J. reine angew. Math. 518 (2000), 187-212. MR.1739404 (2001c:11057)

[4] B.J. Birch, How the number of points of an elliptic curve over a fixed prime field varies, J. London Math. Soc. 43 (1968), 57-60. MR0230682 (37:6242)

[5] S. Frechette, K. Ono, and M. Papanikolas, Gaussian hypergeometric functions and traces of Hecke operators, Int. Math. Res. Not. (2004), no. 60, 3233-3262. MR2096220 (2006a:11055)

[6] J.G. Fuselier, Hypergeometric functions over finite fields and relations to modular forms and elliptic curves, Ph.D. Thesis, Texas A\&M University, 2007. MR 2710790

[7] J.G. Fuselier, Hypergeometric functions over $\mathbb{F}_{p}$ and relations to elliptic curves and modular forms. Proc. Amer. Math. Soc. 138 (2010), no. 1, 109-123. MR2550175 (2011c:11068)

[8] J. Greene, Hypergeometric functions over finite fields, Trans. Amer. Math. Soc. 301 (1987), no. 1, 77-101. MR879564 (88e:11122) 
[9] H. Hijikata, A.K. Pizer, and T.R. Shemanske, The basis problem for modular forms on $\Gamma_{0}(N)$, Mem. Amer. Math. Soc. 82 (1989), no. 418, vi+159 pp. MR960090(90d:11056)

[10] Ihara, Y., Hecke Polynomials as congruence $\zeta$ functions in elliptic modular case, Ann. of Math. (2) 85 (1967), 267-295. MR0207655 (34:7470)

[11] Lennon, C., Gaussian hypergeometric evaluations and traces of Frobenius for elliptic curves, Proc. Amer. Math. Soc. 139 (2011), no. 6, 1931-1938. MR2775369 (2012c:11260)

[12] Lennon, C., A Trace Formula for Certain Hecke Operators and Gaussian Hypergeometric Functions, http://arxiv.org/abs/1003.11578.

[13] J. Riordan, Combinatorial Identities, John Wiley \& Sons, New York, 1968. MR.0231725 $(38: 53)$

[14] R. Schoof, Nonsingular plane cubic curves over finite fields, J. Combin. Theory, Ser. A 46 (1987), no. 2, 183-211. MR914657(88k:14013)

Department of Mathematics and Computer Science, High Point University, High Point, North Carolina 27262

E-mail address: jfuselie@highpoint.edu 